\begin{tabular}{|c|c|c|c|c|}
\hline \multirow[t]{2}{*}{ n (\%) } & \multicolumn{2}{|c|}{$\begin{array}{c}\text { Aged }<70 \text { years } \\
\quad(n=192)\end{array}$} & \multicolumn{2}{|c|}{$\begin{array}{c}\text { Aged } \geq 70 \text { years } \\
(n=87)\end{array}$} \\
\hline & All grades & Grade $\geq 3$ & All grades & Grade $\geq 3$ \\
\hline Any TEAE* & $183(95)$ & $53(28)$ & $84(97)$ & $28(32)$ \\
\hline Nausea & $93(48)$ & 0 & $42(48)$ & $1(1)$ \\
\hline Fatigue/asthenia $^{\dagger}$ & $82(43)$ & $4(2)$ & $41(47)$ & $5(6)$ \\
\hline Anaemia $^{\dagger}$ & $73(38)$ & $26(14)$ & $36(41)$ & $12(14)$ \\
\hline Dysgeusia & $32(17)$ & 0 & $7(8)$ & 0 \\
\hline Vomiting & $28(15)$ & $2(1)$ & $17(20)$ & $1(1)$ \\
\hline Neutropaenia $^{\dagger}$ & $29(15)$ & $2(1)$ & $15(17)$ & $3(3)$ \\
\hline Abdominal pain & $26(14)$ & 0 & $10(11)$ & 0 \\
\hline Diarrhoea & $25(13)$ & 0 & $15(17)$ & 0 \\
\hline Thrombocytopaenia $^{\dagger}$ & $23(12)$ & $2(1)$ & $12(14)$ & $4(5)$ \\
\hline Cough & $22(11)$ & 0 & $7(8)$ & 0 \\
\hline Decreased appetite & $18(9)$ & 0 & $14(16)$ & 0 \\
\hline Arthralgia & $16(8)$ & 0 & $10(11)$ & 0 \\
\hline Urinary tract infection & $13(7)$ & 0 & $14(16)$ & 0 \\
\hline Back pain & $10(5)$ & 0 & $15(17)$ & 0 \\
\hline Serious TEAE & $37(19)$ & - & $18(21)$ & - \\
\hline Dose interruption due to TEAE & $83(43)$ & - & $48(55)$ & - \\
\hline Dose reduction due to TEAE & $38(20)$ & - & $25(29)$ & - \\
\hline Treatment discontinuation due to TEAE & $12(6)$ & - & $9(10)$ & - \\
\hline
\end{tabular}

(median 74.0 years; range 70-85). Among patients aged $<70$ vs $\geq 70$ years, $73 \%$ vs $59 \%$ were Eastern Cooperative Oncology Group (ECOG) performance status 0, 27\% vs 41\% were ECOG performance status $1,64 \%$ vs $49 \%$ had received only two prior platinum regimens, $36 \%$ vs $51 \%$ had received $\geq 3$ prior platinum regimens, and $35 \%$ vs $28 \%$ had a complete response and $64 \%$ vs $70 \%$ had a partial response to their latest platinum regimen. At data cut-off (2 Oct 2020), median PFS and 18-month PFS rates were similar in both age groups (figure 1). Median TFST was 15.6 months (95\% CI 12.218.1 ) in patients aged $<70$ years and 11.4 months $(95 \% \mathrm{CI}$ 9.7-15.6) in patients $\geq 70$ years. The safety profile of maintenance olaparib (median treatment duration 9 months) was generally similar in both age groups (table 1 ).

Conclusion* Efficacy and safety data support the use of maintenance olaparib in non-gBRCAm PSR OC patients irrespective of age.

\section{COST-EFFICIENT ANALYSIS OF BRCANESS STATUS IN HIGH-GRADE SEROUS OVARIAN CARCINOMAS}

${ }^{1}$ E Imyanitov*, 'A Sokolenko, 'T Gorodnova, ${ }^{1}$ I Bizin, ${ }^{1} E$ Kuligina, ${ }^{1} \mathrm{~K}$ Kotiv, ${ }^{1} \mathrm{~A}$ Romanko, ${ }^{1} \mathrm{~T}$ Ermachenkova, ${ }^{1} \mathrm{~A}$ Ivantsov, ${ }^{1} \mathrm{E}$ Preobrazhenskaya, ${ }^{1} \mathrm{~T}$ Sokolova, 'S Aleksakhina, ${ }^{2}$ R Broyde, ${ }^{1}$ I Berlev. ${ }^{1}$ N.N. Petrov Institute of Oncology, St.-Petersburg, Russian Federation; ${ }^{2}$ City Cancer Center, St.-Petersburg, Russian Federation

\subsection{6/ijgc-2021-ESG0.348}

Introduction/Background* Paclitaxel/carboplatin (TCbP) is a standard therapy for high-grade serous ovarian cancer (HGSOC), however many patients do not benefit from this combination. Methodology Genetic profiling was performed for 71 HGSOC consecutive patients, who received neoadjuvant chemotherapy (NACT).

Result(s)* BRCA1/2 germline mutation carriers $(\mathrm{n}=22)$ had longer treatment-free interval (TFI) than non-carriers $(n=49)$ (9.5 vs. 3.8 months; $\mathrm{P}=0.007$ ). $51 \mathrm{HGSOCs}$ with sufficient quality of tumor DNA were examined by the SeqCap EZ CNV/LOH Backbone Design NGS panel, which systematically spans the entire genome at $50 \mathrm{~kb}$ intervals. All 13 tumors obtained from BRCA1/2 germline mutation carriers and 12 sporadic HGSOCs had high number of evenly spread chromosomal breaks, that was defined as a BRCAness phenotype; median TFI for this combined group approached 9.5 months. The remaining 26 HGSOCs had similarly high global $\mathrm{LOH}$ score (above 20\%); however, in contrast to BRCAness tumors, LOH involved large chromosomal segments; these patients had significantly lower TFI (3.7 months; $\mathrm{P}=0.006)$. Comparison between this newly developed BRCAness test, which discriminated tumors simply by the number of affected genomic segments, and the commonly accepted HRD scoring system, revealed high concordance of the results and at least non-inferior clinical performance of our assay. Virtually all tumors with BRCAness (23/25 [92\%]) demonstrated gain at MYC locus, while this event was less common in non-BRCAness HGSOCs (12/26 [46\%]; $\mathrm{P}=0.0006)$. All patients with CCNE1 amplification ( $\mathrm{n}=7$ ), TP53 R175H substitution ( $\mathrm{n}$ $=6)$, and RB1 mutation $(n=4)$ had poor response to TCbP. Conclusion* BRCA1/2 germ-line testing has superior performance in identifying responders to TCbP. Simple and rapid PCR-based tests for MYC and CCNE1 amplification allow to classify patients for potential responders and non-responders with a reasonable level of accuracy. BRCAness phenotype can be reliably detected by a laboratory-scale NGS assay, which evaluates the total number of chromosomal breaks. It is of concern that TCbP is being routinely administered both to potential responders and to potential non-responders to this scheme. Novel treatment options for the latter category of HGSOC patients need to be searched within preclinical and clinical studies.

\section{EFFICACY OF NIRAPARIB BY TIMING OF SURGERY AND RESIDUAL DISEASE: A POST-HOC ANALYSIS OF PATIENTS IN THE PRIMA/ENGOT-OV26/GOG-3012 STUDY}

${ }^{1} \mathrm{R}$ O'cearbhaill*, ${ }^{2} \mathrm{JA}$ Pérez-Fidalgo, ${ }^{3} \mathrm{BJ}$ Monk, ${ }^{4} \mathrm{C}$ Vulsteke, ${ }^{5} \mathrm{C}$ Mccormick, ${ }^{6} \mathrm{~S}$ Hietanen, ${ }^{7} \mathrm{RG}$ Moore, ${ }^{8} \mathrm{G}$ Artioli, ${ }^{9} \mathrm{MS}$ Shahin, ${ }^{10} \mathrm{~F}$ Selle, ${ }^{11} \mathrm{WH}$ Bradley, ${ }^{12} \mathrm{~K}$ Baumann ${ }^{*},{ }^{13} \mathrm{D}$ O'malley, ${ }^{14}$ I Tusquets, ${ }^{15} \mathrm{BM}$ Slomovitz, ${ }^{16} \mathrm{~T}$ Levy, ${ }^{17} \mathrm{~F}$ Joly, ${ }^{18}$ I Malinowska, ${ }^{18} \mathrm{D}$ Gupta, ${ }^{19} \mathrm{~A}$ GonzálezMartin. ${ }^{1}$ GOG and the Department of Medicine, Memorial Sloan Kettering Cancer Center, Weill Cornell Medical College, New York, NY, USA; ${ }^{2}$ Department of Medical Oncology, INCLIVA University Hospital of Valencia, CIBERONC, Valencia, Spain; ${ }^{3}$ Arizona Oncology (US Oncology Network), University of Arizona College of Medicine, Creighton University School of Medicine, Phoenix, AZ, USA; ${ }^{4} B G O G$ and the Department of Medical Oncology and Hematology, AZ Maria Middelares, Ghent, Belgium, and the Department of Molecular Imaging, Pathology, Radiotherapy, and Oncology, Center for Oncological Research, Antwerp University, Antwerp, Belgium; ${ }^{5}$ GOG and Legacy Medical Group Gynecologic Oncology, Portland, OR, USA; ${ }^{6}$ Department of Obstetrics and Gynecology, Turku University Hospital, Turku, Finland; 'Division of Gynecologic Oncology, Wilmot Cancer Institute, Department of Obstetrics and Gynecology, University of Rochester, Rochester, NY, USA; ${ }^{8}$ ULSS 3 Serenissima, U.O.C. Oncologia ed Ematologia Oncologica, Mirano, Venice, Italy; ${ }^{9}$ Abington Hospital-Jefferson Health, Sidney Kimmel Cancer Center of Thomas Jefferson University, Willow Grove, PA, USA; ${ }^{10}$ GINECO and Groupe Hospitalier Diaconesses-Croix Saint Simon, Paris, France; ${ }^{11} \mathrm{GOG}$ and the Department of Obstetrics and Gynecology, Medical College of Wisconsin, Milwaukee, WI, USA; ${ }^{12}$ Arbeitsgemeinschaft Gynäkologische Onkologie and the Department of Gynecology and Obstetrics, Klinikum der Stadt Ludwigshafen, Ludwigshafen, Germany; ${ }^{13}$ Ohio State University, James Comprehensive Cancer Center, Columbus, $\mathrm{OH}$, USA; ${ }^{14}$ Medical Oncology Department, Hospital del Mar, Barcelona, Spain; ${ }^{15}$ Broward Health, Florida International University Wertheim College of Medicine, Miami, FL, USA; ${ }^{16}$ Department of Obstetrics and Gynecology, Wolfson Medical Center, Sackler School of Medicine, Tel Aviv University, Holon, Israel; ${ }^{17}$ Medical Oncology Department, Centre Francois Baclesse, Caen, France; ${ }^{18} \mathrm{GlaxoSmithKline,} \mathrm{Waltham,} \mathrm{MA,} \mathrm{USA;}{ }^{19}$ Grupo Español de Investigación en Cáncer de Ovario (GEICO) and Medical Oncology Department, Clínica Universidad de Navarra, Madrid, Spain

10.1136/ijgc-2021-ESG0.349 